\title{
Quantitative Analysis of Vessel Permeability in Mouse Endometrial Implants
}

\author{
Niraj Trivedi*, Glen DeLoid**, Renee Dickie**, Christian M. Becker*, Jean Lai**, Patricia \\ Iglesias***, Stephanie Chirico*, Tae Funakoshi*, Robert J. D’Amato*, Rick A. Rogers***, \\ and Maria A. Rupnick* \\ *Department of Vascular Biology, Children's Hospital Boston, Karp Family Research \\ Building, Boston, MA 02215 \\ **Biomedical Imaging Laboratory, Harvard School of Public Health, 665 Huntington Ave, \\ Boston, MA 02115 \\ ***UANL Medical School, Monterrey, Mexico
}

Estimates of vessel leakiness are valuable in assessing the effect of anti-angiogenic drugs on vascular permeability. Here we present image processing methods to quantify vessel permeability to albumin in whole mount or thick section specimens.

Mice with endometrial implants in the peritoneal wall [1,2] were euthanized 7 days postimplantation and injected I.V. with FITC-conjugated albumin. Nine of the mice had been treated with an angiogenesis inhibitor and eight mice were untreated controls. Vessels were imaged using confocal microscopy (Figs. 1, 2).

To identify albumin filled vessels serial sections were globally thresholded to distinguish background from albumin. Pixels were categorized as belonging to vessel contents if they were within an apparent vessel with a minimum width of 5-8 microns in any direction, independent of length and vessel morphology.

To determine the permeability coefficient for vessels, 12 test line segments were applied passing through the vessel center in different orientations (Fig 1). An exponential decay curve fit was performed on a histogram of intensity vs. distance (Fig 3) along each test segment. The exponential decay curve is in the form of $\mathrm{e}^{(-\mathrm{x} / \tau)}$, where $\mathrm{x}$ is the distance from the vessel boundary to the free albumin, and $\tau$ is the lifetime decay, referred to in this study as the permeability coefficient. Larger coefficients correspond to greater amounts of free albumin present outside the vessel wall, indicating higher vessel permeability. The highest and lowest permeability coefficients were excluded for each image section analyzed. The permeability coefficients of the treated and untreated groups were then averaged.

For comparison, 3-D connectivity algorithm was used to distinguish between intravascular and leaked albumin pixels. Pixels above threshold that were connected to a minimum number of voxels (corresponding to a minimum width of 5-8 microns in any direction) of above-threshold pixels in $\mathrm{x}, \mathrm{y}$, and $\mathrm{z}$ were considered to represent vessel contents (VC). All other pixels above threshold were counted as free albumin (FA). The mean ratio of free albumin to vessel contents pixels $(\mathrm{FA} / \mathrm{VC})$ was calculated. 
From the 2-D analysis of serial section data, the mean permeability coefficients were 19.1 and 13.2 for vessels in the treated and untreated groups, respectively $(p<0.001)$. Analysis of the 3$\mathrm{D}$ connectivity resulted in mean FA/VC values of 1.57 and 0.41 for vessels in the treated and untreated groups, respectively, but with a high variance, and non-significant difference $(p=0.39)$.

These results suggest that the permeability coefficient is a more sensitive indicator of vascular permeability of labeled albumin than $3-\mathrm{D}$ connectivity analysis.

\section{References}

[1] A.M. Cummings and J.L. Metcalf, Reprod Toxicol. 9 (1995) 233.

[2] M.W.Vernon and E.A. Wilson, Fertil Steril. 44 (1985) 684.

[3] This research was supported by NIH PO1 CA 45548, NIH HL 65960, and NIH ES 00002.

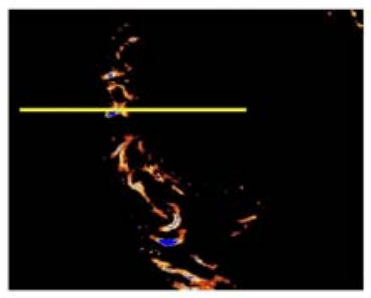

A

Figure 1. FITC-albumin perfused vessels from an untreated mouse (A) and a mouse treated with an anti-angiogenic drug (B). Test line in yellow.
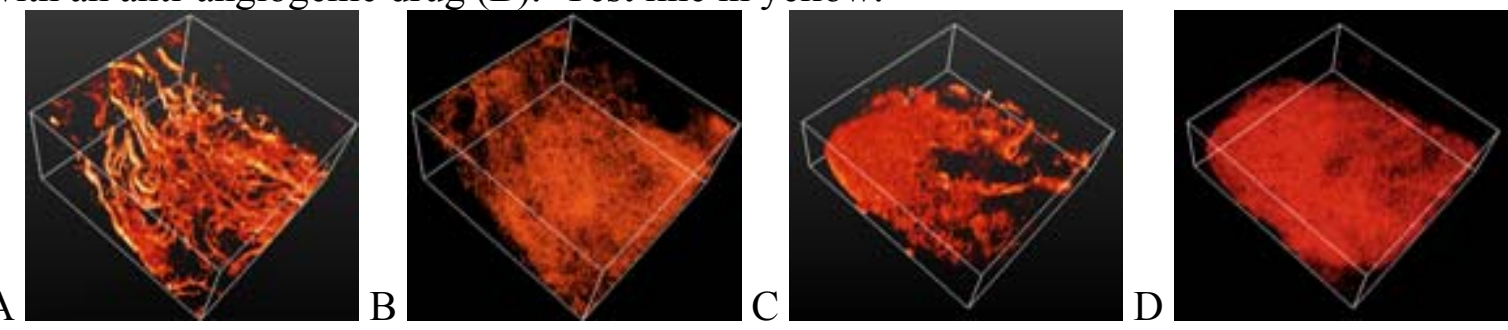

Figure 2. 3-D reconstructions of vessel $(A, C)$ and leaked albumin $(B, D)$ in untreated $(A, B)$ and treated $(\mathrm{C}, \mathrm{D})$ endometrial implants.
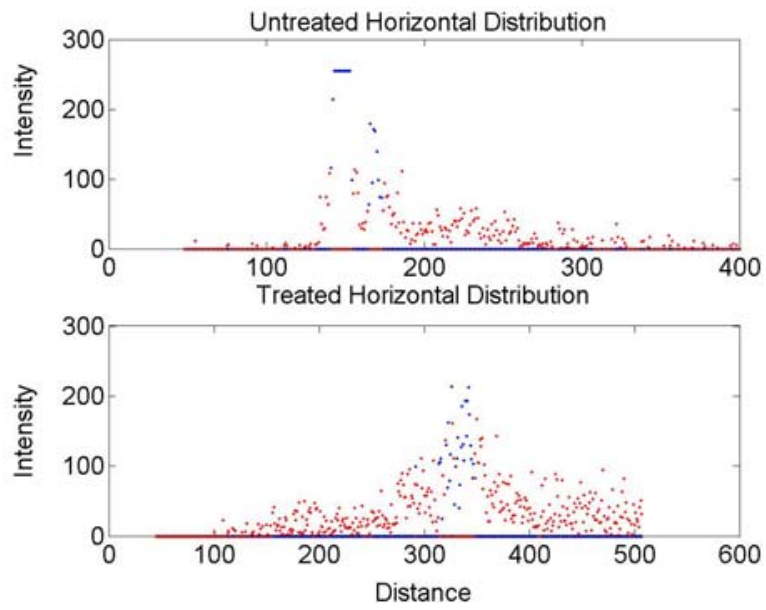

Figure 3. Line distribution taken from test lines passing through vessels in Untreated (top), and Treated (bottom) in Figure 1 where $\mathrm{x}$-axis is distance in pixels and y-axis is intensity of FITC-albumin. Blue points represent vessel and red points represent free albumin. 